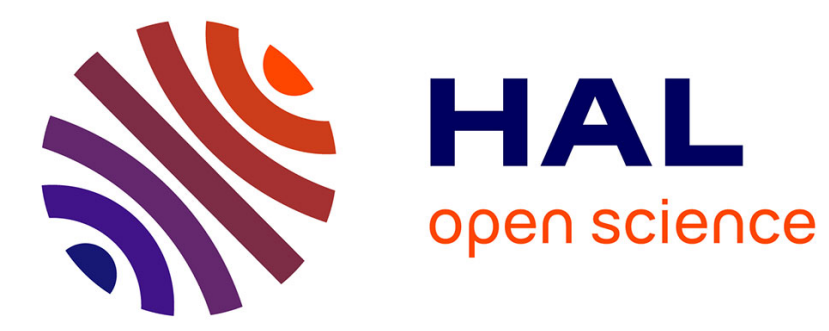

\title{
Mechanical Equivalent of Quantum Heat Engines
}

\author{
Jacques Arnaud, Laurent Chusseau, Fabrice Philippe
}

\section{To cite this version:}

Jacques Arnaud, Laurent Chusseau, Fabrice Philippe. Mechanical Equivalent of Quantum Heat Engines. Physical Review E: Statistical, Nonlinear, and Soft Matter Physics, 2008, 77, pp.061102. 10.1103/PhysRevE.77.061102 . lirmm-00363624

\section{HAL Id: lirmm-00363624 https://hal-lirmm.ccsd.cnrs.fr/lirmm-00363624}

Submitted on 11 Jun 2021

HAL is a multi-disciplinary open access archive for the deposit and dissemination of scientific research documents, whether they are published or not. The documents may come from teaching and research institutions in France or abroad, or from public or private research centers.
L'archive ouverte pluridisciplinaire HAL, est destinée au dépôt et à la diffusion de documents scientifiques de niveau recherche, publiés ou non, émanant des établissements d'enseignement et de recherche français ou étrangers, des laboratoires publics ou privés. 


\title{
Mechanical equivalent of quantum heat engines
}

\author{
Jacques Arnaud* \\ Mas Liron, F30440 Saint Martial, France
}

Laurent Chusseau ${ }^{\dagger}$

Institut d'Électronique du Sud, UMR 5214 CNRS, Université Montpellier II, F34095 Montpellier, France

\author{
Fabrice Philippe \\ Laboratoire d'Informatique de Robotique et de Microélectronique de Montpellier, UMR 5506 CNRS, 161 Rue Ada, \\ F34392 Montpellier, France
}

(Received 22 January 2008; published 3 June 2008)

\begin{abstract}
Quantum heat engines employ as working agents multilevel systems instead of classical gases. We show that under some conditions quantum heat engines are equivalent to a series of reservoirs at different altitudes containing balls of various weights. A cycle consists of picking up at random a ball from one reservoir and carrying it to the next, thereby performing or absorbing some work. In particular, quantum heat engines, employing two-level atoms as working agents, are modeled by reservoirs containing balls of weight 0 or 1 . The mechanical model helps us prove that the maximum efficiency of quantum heat engines is the Carnot efficiency. Heat pumps and negative temperatures are considered.
\end{abstract}

DOI: 10.1103/PhysRevE.77.061102

PACS number(s): 05.30.-d, 07.20.Pe

\section{INTRODUCTION}

Quantum heat engines employ multilevel systems (quantized free particles in a box, three-level atoms, quantized harmonic oscillators, or electrons immersed in a magnetic field) as working agents instead of classical gases. The operation of such quantum heat engines has been treated by Geva and Kosloff [1], Feldmann et al. [2], Opatrny and Scully [3], and He et al. [4]. Advanced papers on quantum heat engines $[1,3,4]$ are often concerned with maximizing powers. We restrict ourselves here to the work performed over slow cycles. A related discussion is given by Kieu [5]. The recent detailed discussion by Quan et al. [6] contains a good list of references to which the reader is referred for a more complete historical account.

We consider particularly two-level agents such as electrons immersed in magnetic fields $B$. In that case the electrons may reside in either one of two energy levels separated by $\epsilon \propto B$ or, in appropriate units, $\epsilon=B$. In the conventional form of heat engines, a "working agent" containing electrons may be in contact with a thermal bath and be submitted to some $\epsilon$ value. The so-called "Otto cycle" consists of putting the working agent in contact with a hot bath and submitting it to some large $\epsilon$. The working agent is then isolated from the hot bath, put in contact with a cold bath, and submitted to some small $\epsilon$ value. Finally, the working agent returns to the hot bath, thereby closing the cycle. The Otto cycle just described is not optimum as far as efficiency is concerned, but we consider it initially for the sake of simplicity (in a more general configuration, $\epsilon$ varies also while the working agent is in contact with the hot and cold baths).

\footnotetext{
*arnaudj2@wanadoo.fr

†chusseau@univ-montp2.fr

†Also at MIAp, Université Paul Valéry, F34199 Montpellier, France; philippe@lirmm.fr
}

Our model differs slightly from the conventional one just described. Work is produced when a randomly selected electron is carried from a high-magnetic-field high-temperature region into a low-magnetic-field low-temperature region, and conversely. That work is delivered to the source of the field that carries the electrons from one region to the other, e.g., an electrical battery.

Note that heat engines operate with (hot and cold) reservoirs that are so large that their temperatures are negligibly affected by the energy-delivering cycles. One may consider instead an ensemble of engines that are initially all in the same state. The work performed over a cycle by one member of the ensemble may slightly differ from the work performed by another member of the ensemble. But only average energies are presently considered.

On the historical side, it is worthwhile recalling that, contrary to what some authors have said in the past, the theory of classical heat engines and heat pumps established by Carnot around 1824 is entirely accurate. The first law of thermodynamics was expressed by Carnot in those terms: "Heat is nothing but motive power, or rather another form of motion. Wherever motive power is destroyed, heat is generated in precise proportion to the quantity of motive power destroyed; conversely, wherever heat is destroyed, motive power is generated" $[7,8]$. Carnot also gave an accurate correspondence between a calorie (the amount of heat required to raise the temperature of one gram of water by $1^{\circ} \mathrm{C}$ ) and the energy required to raise a mass of one kilogram by one meter in the earth gravitational field. On the other hand, it is an empirical fact that heat may flow spontaneously from hot to cold bodies, but that the converse never occurs. This fact is accounted for by introducing the concept of entropy, called calorique by Carnot: "Carnot used chaleur when referring to heat in general, but when referring to the motive power of fire that is brought about when heat enters an engine at high temperature and leaves at low temperature, he uses the expression chute de calorique, never chute de chaleur. Carnot 
had in the back of his mind the concept of entropy, for which he reserved the term calorique" $[9,10]$.

The efficiency $\eta$ of a heat engine is defined as the ratio of the work $W$ performed and the high-temperature-reservoir heat consumption $-Q_{h}$, both being expressed in the same energy unit, e.g., in joules. Carnot found that heat engines reach their maximum efficiency when they are reversible. The famous formula $\eta_{C} \equiv \eta_{\max }=1-T_{l} / T_{h}$, where $T_{l}$ and $T_{h}$ denote the cold and hot reservoirs absolute temperatures, respectively, follows from his work. The work performed is then $W=\left(T_{h}-T_{l}\right) S$, where $S$ denotes the entropy transferred from the hot to the cold bath. Carnot gave approximate expressions for $T_{h}, T_{l}$, and $S$ on the basis of experimental results known at his time.

If we introduce the inverse absolute temperatures $\beta$, the Carnot formula reads

$$
\eta_{C}=1-\frac{\beta_{h}}{\beta_{l}}, \quad \beta_{l}>\beta_{h}>0 .
$$

The cases where one of the two heat reservoirs, or both, have negative temperatures will be considered. Observation of negative temperatures was first reported in 1951 by Purcell and Pound, (see, e.g., [[11], p.102]). In the case of electrons submitted to a magnetic field, negative temperatures are obtained by suddenly reversing the magnetic-field direction. In our mechanical model described below, it suffices to select a number of weight- 1 balls that exceed half the total number of balls. The operation of heat engines and heat pumps with negative reservoir temperatures has been adequately treated by $[12]$.

The purpose of this paper is to show that quantum heat engines are equivalent to purely mechanical engines akin to water mills. Specifically, we consider a series of reservoirs at different altitudes in the Earth's gravitational field, containing balls of different weights. A cycle consists of picking up at random a ball from one reservoir and carrying it to the next, thereby performing or absorbing some work. In particular, quantum heat engines that employ as working agent electrons submitted to magnetic fields are modeled by reservoirs containing balls of weight 0 or 1 . The efficiency of our mechanical engine is defined as the ratio of the work $W$ performed to the work that would be performed if the highreservoir content were dropped all the way down to some lower reference level. This efficiency is less than unity even under ideal conditions.

As said earlier, the heat engine that we consider is labeled "quantum" because the working agent may reside in only a few discrete energy levels, typically 0 and 1 . The Planck constant does not enter in our discussion because the parameter controlling the working agent is an energy $\epsilon$ determined from the outside. In contradistinction, in the case of oscillators (see, e.g., [13]), the externally controlled parameter is not directly the energy but instead the oscillator angular frequency $\omega$. In that case the Planck constant divided by $2 \pi, \hbar$, enters through the expression of the elementary oscillator energy according to the Planck-Einstein formula, $\epsilon=\hbar \omega$. Questions relating to the coherence of the wave function do not enter in our discussion.
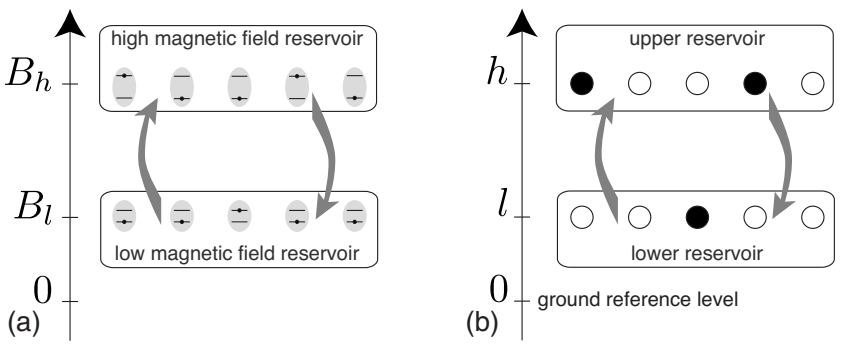

FIG. 1. (a) Schematic representation of a quantum heat engine employing electrons submitted to a magnetic field of intensity $B$ as working agents. Electrons are shown as two-level systems in either their upper or lower state of energy. The lower-level energy is set at zero, and the upper-level energy is denoted $\epsilon=B$, in appropriate units. We consider two reservoirs containing $N=5$ electrons each. In the $l$ reservoir there is a single electron in the upper state, while in the $h$ reservoir two electrons are in the upper state. In (b) electrons in their low-energy level are represented by balls of weight 0 (open circles), while electrons in their high-energy levels are represented by balls of weight 1 (black-filled circles) of potential energy $\epsilon$ that equals the altitude. In both (a) and (b) a cycle consists of picking up at random an electron (respectively, a ball) from a reservoir and carrying it to the next. This operation preserves the total number of balls in each reservoir, but some work is being performed (heat engine, the case of the figure) or absorbed (heat pump).

The statistical properties of the mechanical engines considered follow from a simple urn model. A reservoir (or urn) contains $n$ balls of weight 1 and $N-n$ balls of weight 0 . The probability of picking up any particular ball is equal to $1 / \mathrm{N}$. It is, of course, implied that once a ball has been introduced in the reservoir or removed from it some stirring occurs and sufficient time is allowed before a new ball is being picked up or added, as is the case for lottery machines. The average ball weight $n / N$ will be called "force" and denoted $f$. Although the total number of balls shown in Fig. 1 is $N=5$ for the sake of clarity, it is implied in the theory that $N$ and $n$ are large numbers, so that the rational number $f$ may be viewed as a real number.

To our knowledge, the model considered in the present paper relating mechanical engines to thermodynamics cycles [14] has not been published before in the open literature. It provides a feel for the method of extraction of mechanical energy from heat baths using only elementary concepts. The purely mechanical device presently discussed could have been constructed in ancient times, with macroscopic balls (with a mass $\approx 0.1 \mathrm{~kg}$ ) in a series of lottery-type containers. The only theoretical knowledge required to evaluate the average work $W$ performed by such a mechanism is the concept of potential energy, and the fact that the probability of picking up a particular ball among a collection of $N$ similar balls is equal to $1 / N$. Fluctuations of the work produced could be evaluated on the same basis, but this is not done in the present paper.

In Sec. II we consider Otto engines. In Otto heat engines, the parameter (e.g., the length of a gas-filled cylinder for a classical engine, or the magnetic field for the electron quantum heat engine) does not vary when the working material is in contact with a heat bath. Our mechanical model in that case consists of two reservoirs containing $N$ balls each, at 
altitudes $\epsilon_{l}$ and $\epsilon_{h}$, respectively. In Sec. III we consider $2 m$ reservoirs, split into a series of $m$ high reservoirs, and a series of $m$ low reservoirs. We show in Sec. IV that in the $m \rightarrow \infty$ limit and appropriate parameter values, the efficiency and work performed are given by the Carnot expressions quoted earlier. Our reservoir parameters (altitudes and forces) are related to statistical mechanical concepts of temperature and entropy in Sec. V. Finally, in Sec. VI, an application is made to quantum heat engines and heat pumps.

\section{OTTO CYCLE}

Figure 1 represents in (a) a quantum heat engine that employ as working agent electrons immersed in magnetic fields $B_{l}$ or $B_{h}$, and in (b) the equivalent mechanical model consisting of balls of weight $0 \mathrm{~N}$ (white circles) or $1 \mathrm{~N}$ (black circles). On the left (low reservoir) the weight- 1 balls at altitude $\epsilon_{l}=1 \mathrm{~m}$ have potential energy $1 \mathrm{~J}$. On the right (high reservoir), weight- 1 balls at altitude $\epsilon_{h}=2 \mathrm{~m}$ have potential energy $2 \mathrm{~J}$. Of course, weight-0 balls have no energy whatever their height.

The low reservoir contains four balls of weight 0 and one ball of weight 1 , so that the average weight, or force $f_{l}$ $=1 / 5$. The high reservoir contains three balls of weight 0 and two balls of weight 1 , so that the average force $f_{h}=2 / 5$. Clearly, the ball model exhibits the same level energies as the electronic system, except perhaps for a scale factor. If the ball system is put in contact with a heat bath, the latter is supposed to be able to exchange balls with the system, so that temperatures eventually equalize.

More generally, the two reservoirs contain $N \gg 1$ balls each. The number of balls of weight 1 in the reservoir at level $\epsilon_{h}$ is denoted by $n_{h}$, while the number of balls of weight 1 in the reservoir at level $\epsilon_{l}$ is denoted by $n_{l}$. We define forces (or average ball weights) as $f_{h} \equiv n_{h} / N, f_{l} \equiv n_{l} / N$. According to classical mechanics, when a ball of weight 1 is added to a reservoir at altitude $\epsilon$ the reservoir potential energy is incremented by $\epsilon$. On the other hand, the probability of picking up any particular ball from a reservoir is $1 / N$. Accordingly, when a randomly selected ball is removed from a reservoir at altitude $\epsilon$ the reservoir average potential energy is reduced by $\epsilon f$. From now on, increments in reservoirs potential energies are labeled by the letter $Q$, anticipating a correspondence with heat.

We first consider Otto cycles because they involve only two reservoirs. We also consider only two energy levels. In our mechanical model, a cycle consists of transferring a randomly selected ball from the high reservoir to the lower one, while a ball randomly selected from the low reservoir is being transferred to the higher one. Note that the total number of balls in each reservoir remains $N$ (this ball exchange is symbolized in Fig. 1 by arrows).

In conventional classical or quantum heat engines, a single working agent is considered (for example, a gas- or atom-filled cylinder) repeatedly displaced between hot and cold baths. Presently, we are instead considering a sequence of working agents (called "reservoirs"). Further, instead of displacing reservoirs as a whole, this is their content which is being displaced at random. Because only average works are

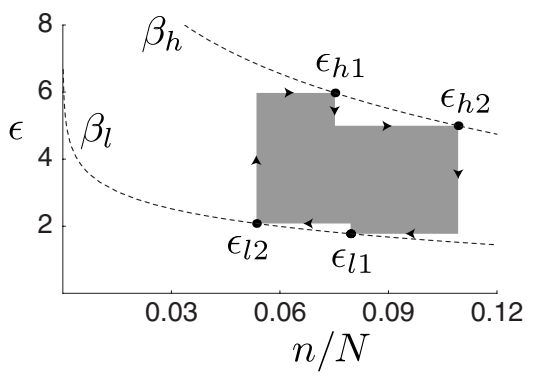

FIG. 2. This figure represents the $\epsilon(f)$ diagram of a mechanical device involving two high reservoirs ( $h$ subscripts) and two low reservoirs ( $l$ subscripts). The four reservoirs are shown as dots with $f \equiv n / N$ in the abscissa, where $n$ represents the average number of balls of weight 1 in the reservoir. The work performed per cycle, $W \approx 0.193 \mathrm{~J}$, is equal to the shaded area, and the efficiency $\eta$ $\approx 0.643$ is the maximum possible value under the present conditions.

being considered (and not work fluctuations), the mechanism considered in the present paper is effectively the same as the conventional one, as we shall see. Accordingly, the reader should not be disturbed by the fact that the random ball displacement mechanism discussed below seems at first to differ from the conventional heat-engine mechanism.

Upon the exchange of randomly selected balls between the two reservoirs, the energies added to the low and high reservoirs read, respectively,

$$
\begin{gathered}
Q_{l}=\epsilon_{l}\left(f_{h}-f_{l}\right), \\
Q_{h}=\epsilon_{h}\left(f_{l}-f_{h}\right) .
\end{gathered}
$$

The work performed follows from the law of conservation of energy $W+Q_{l}+Q_{h}=0$; that is,

$$
W=-Q_{l}-Q_{h}=\left(\epsilon_{h}-\epsilon_{l}\right)\left(f_{h}-f_{l}\right) .
$$

The engine efficiency is defined as the ratio of the work performed $W$ and the energy $-Q_{h}$ lost by the higher reservoir; that is,

$$
\eta \equiv \frac{W}{-Q_{h}}=1-\frac{\epsilon_{l}}{\epsilon_{h}} .
$$

For example, if $\epsilon_{l}=1 \mathrm{~m}, \epsilon_{h}=2 \mathrm{~m}$, the engine efficiency $\eta$ $=0.5$. For the force values shown in the figure, the work performed $W=(2-1)(2 / 5-1 / 5) \mathrm{J}=0.2 \mathrm{~J}$.

The identification of efficiency in Eq. (4) with that of a classical Otto cycle has been discussed by Quan et al. [6]. Readers are referred to their work.

\section{III. $\boldsymbol{m}$ SUBRESERVOIRS}

Let us consider first two low reservoirs (referred to as low subreservoirs) at altitudes $\epsilon_{l_{1}}, \epsilon_{l_{2}}$, with forces $f_{l_{1}}, f_{l_{2}}$, respectively, and two high subreservoirs at altitudes $\epsilon_{h_{1}}, \epsilon_{h_{2}}$, with forces $f_{h_{1}}, f_{h_{2}}$, respectively (see Fig. 2), where the forces $f$ are shown on the horizontal axis and the altitudes $\epsilon$ on the vertical axis.

Consider the exchange of a randomly picked ball from subreservoir $l_{1}$ to subreservoir $l_{2}$, from subreservoir $l_{2}$ to sub- 
reservoir $h_{1}$, from subreservoir $h_{1}$ to subreservoir $h_{2}$, and finally, from subreservoir $h_{2}$ to subreservoir $l_{1}$, thereby closing the cycle indicated by an arrow on the figure. Using the same argument as in the previous section we evaluate the total potential energy $Q_{l}$ added to the two low reservoirs, and the total potential energy $Q_{h}$ added to the two high reservoirs

$$
\begin{gathered}
Q_{l}=\epsilon_{l_{1}}\left(f_{h_{2}}-f_{l_{1}}\right)+\epsilon_{l_{2}}\left(f_{l_{1}}-f_{l_{2}}\right), \\
Q_{h}=\epsilon_{h_{1}}\left(f_{l_{2}}-f_{h_{1}}\right)+\epsilon_{h_{2}}\left(f_{h_{1}}-f_{h_{2}}\right) .
\end{gathered}
$$

Referring to the closed path in Fig. $2,-Q_{h}$ is the area under the upper line, and $Q_{l}$ is the area under the lower line, so that the work performed $W=-Q_{h}-Q_{l}$ is the shaded area on the figure.

More generally for $m$ low subreservoirs and $m$ high subreservoirs, we have

$$
\begin{gathered}
Q_{l}=\epsilon_{l_{1}} f_{h_{m}}+\sum_{i=1}^{m-1} f_{l_{i}}\left(\epsilon_{l_{i+1}}-\epsilon_{l_{i}}\right)-\epsilon_{l_{m}} f_{l_{m}}, \\
Q_{h}=\epsilon_{h_{1}} f_{l_{m}}+\sum_{i=1}^{m-1} f_{h_{i}}\left(\epsilon_{h_{i+1}}-\epsilon_{h_{i}}\right)-\epsilon_{h_{m}} f_{h_{m}} .
\end{gathered}
$$

Note that the expressions for $Q_{l}$ and $Q_{h}$ given above are the same provided the labels $l, h$ are interchanged. The expressions for the total work performed $W$ and the efficiency $\eta$ follow from the expressions of $Q_{l}$ and $Q_{h}$ given above.

\section{CARNOT CYCLE}

We considered above subreservoirs with arbitrary forces $f_{i}$ and altitudes $\epsilon_{i}$. We now restrict the generality by supposing that the forces depend on the reservoir altitudes according to laws of the form

$$
f_{l_{i}}=f\left(\beta_{l} \epsilon_{l_{i}}\right), \quad f_{h_{i}}=f\left(\beta_{h} \epsilon_{h_{i}}\right), \quad i=1,2 \ldots m,
$$

where $\beta_{l} \equiv 1 / T_{l}$ and $\beta_{h} \equiv 1 / T_{h}$ denote two arbitrary numbers, and $f()$ denotes an arbitrary function of its argument, to be specified later on. Under that restriction, the above expressions for the reservoir energy increments read, according to Eqs. (6) and (7),

$$
\begin{gathered}
\beta_{l} Q_{l}=L_{1} f\left(H_{m}\right)+\sum_{i=1}^{m-1} f\left(L_{i}\right)\left(L_{i+1}-L_{i}\right)-L_{m} f\left(L_{m}\right), \\
\beta_{h} Q_{h}=H_{1} f\left(L_{m}\right)+\sum_{i=1}^{m-1} f\left(H_{i}\right)\left(H_{i+1}-H_{i}\right)-H_{m} f\left(H_{m}\right),
\end{gathered}
$$

where we have defined $L_{i}=\beta_{l} \epsilon_{l_{i}}$ and $H_{i}=\beta_{h} \epsilon_{h_{i}}, \quad i$ $=1,2, \ldots, m$.

If the $\epsilon$ values do not vary much from one low subreservoir to the next, and likewise, do not vary much from one high subreservoir to the next, sums may be replaced by integrals and we have

$$
\begin{aligned}
& \beta_{l} Q_{l}=s\left(L_{1}, H_{m}\right)-s\left(L_{m}\right), \\
& \beta_{h} Q_{h}=s\left(H_{1}, L_{m}\right)-s\left(H_{m}\right),
\end{aligned}
$$

where

$$
s(x, y) \equiv x f(y)-\int^{x} f\left(x^{\prime}\right) d x^{\prime}, \quad s(x) \equiv s(x, x),
$$

the lower limit of the integral being unimportant.

When $L_{1}=H_{m}$ and $H_{1}=L_{m}$ the quantity $\beta_{l} Q_{l}+\beta_{h} Q_{h}$ vanishes and the maximum efficiency

$$
\eta=1-\frac{T_{l}}{T_{h}}
$$

is reached. The work performed $W=-Q_{l}-Q_{h}$ is the product of $T_{h}-T_{l}$ and the change of $s$, namely,

$$
W=\left(T_{h}-T_{l}\right) S, \quad S \equiv s\left(L_{1}\right)-s\left(H_{1}\right),
$$

where the $s($ ) functions are defined in Eq. (10). These expressions for the work and efficiency are formally the same as those applicable to a reversible heat engine.

\section{STATISTICAL MECHANICS AND THE $\boldsymbol{f}$ FUNCTION}

The main concept of statistical mechanics is that states of isolated systems corresponding to the same energy are equally likely to occur. The degeneracy (or statistical weight) $\mathcal{W}$ is the number of distinguishable states. Let $N$ denote the total number of balls in a reservoir at altitude $\epsilon$ in the Earth's gravitational field and $n$ the number of balls of weight 1 , the other balls being weightless [14]. The potential energy is clearly $U=n \epsilon$. On the other hand, we have (see, e.g., $[11,15]$ )

$$
\mathcal{W}(n)=\frac{N !}{n !(N-n) !} .
$$

For $N=2$, for example, the degeneracy $\mathcal{W}(1)=2$ because either one of the two balls may have weight 1 .

The entropy is defined from the statistical mechanical point of view as: $S(n)=\ln [\mathcal{W}(n)]$, the Boltzmann constant being set equal to unity. The inverse temperature is further defined as $\beta \equiv d S / d U \equiv(1 / \epsilon) d \ln [\mathcal{W}(n)] / d n$. The derivative cannot be evaluated exactly since $n$ is an integer. The best one can do is to apply the Stirling approximation of factorials: $d \ln (n !) / d n \approx \ln (n !)-\ln [(n-1) !]=\ln (n)$. We obtain from Eq. (13),

$$
\beta \epsilon=\ln \left(\frac{N}{n}-1\right) \Leftrightarrow f=\frac{1}{\exp (\beta \epsilon)+1} .
$$

The reservoir temperature is positive when $n<N / 2$ and negative when $n>N / 2$. For two states, the $f()$ function introduced in Eq. (7) is therefore $f(x)=1 /[\exp (x)+1]$, from which the $s(x, y)$ function introduced in Eq. (10) follows from a simple integration.

The mechanical equivalent of an atom with $q$ evenly spaced states is a reservoir containing balls with $q$ evenly spaced weights. A discussion about multilevel quantum Otto heat engines can be found in [16]. For $q$ evenly spaced states, 
Eq. (14) straightforwardly generalizes using similar reasoning,

$$
f(x)=\frac{1}{\exp (x)-1}-\frac{q}{\exp (q x)-1},
$$

which reduces to the previous expression if $q=2$ and to the expression applicable to harmonic oscillators when $q \rightarrow \infty$ [13], except for an arbitrary additive constant.

The two-argument entropy defined in Eq. (10) reads

$$
\begin{gathered}
s(x, y)=S(x, y)-S(q x, q y), \\
S(x, y) \equiv \frac{x}{\exp (y)-1}-\ln [1-\exp (-x)] .
\end{gathered}
$$

From a previous expression of the work performed, it follows that the maximum work in a Carnot cycle is $W_{\max }$ $=\left(T_{h}-T_{l}\right) \ln (q)$.

For the sake of comparison with previous works, note that the quantity denoted here, $f \equiv n / N$, corresponds to the electron spin (divided by $\hbar$ ) plus $1 / 2$. The magnetic field $\epsilon$ corresponds to the quantity denoted by $\omega$ in [1]. The relation between $n / N$ and the inverse temperature $\beta$ given later on in Eq. (17) coincides with the one given in that reference.

\section{APPLICATIONS}

For two-level systems the reservoirs inverse temperatures are, according to Eq. (14),

$$
\beta_{l}=\frac{\ln \left(\frac{N}{n_{l}}-1\right)}{\epsilon_{l}}, \quad \beta_{h}=\frac{\ln \left(\frac{N}{n_{h}}-1\right)}{\epsilon_{h}},
$$

respectively. Note that $\beta$ is positive when $n$ is smaller than $N / 2$ and negative when $n$ is larger than $N / 2$.

Positive temperatures. When the two reservoirs have positive temperatures the maximum efficiency given in Eq. (1),

$$
\eta_{C}=1-\frac{\beta_{h}}{\beta_{l}}=1-\frac{\epsilon_{l}}{\epsilon_{h}} \ln \left(\frac{N / n_{h}-1}{N / n_{l}-1}\right),
$$

always exceeds the Otto-cycle efficiency $\eta=1-\left(\epsilon_{l} / \epsilon_{h}\right)$ since $n_{h}>n_{l}$ when the work produced is positive.

As an example of a heat engine, suppose that $\epsilon_{l}=1 \mathrm{~m}$ and $\epsilon_{h}=2 \mathrm{~m}$. The calculated efficiency for an Otto cycle is $\eta$ $=0.5$. Further, for $N=10000, n_{l}=2000$, and $n_{h}=3000$, the work delivered per cycle is $W=0.1 \mathrm{~J}$. For the above numerical values, we calculate that $\beta_{l}=1.38, \beta_{h}=0.42$, and therefore the maximum (Carnot) efficiency $\eta_{C}=0.69$. The shaded areas in Fig. 3 are obtained numerically by randomly selecting positive $\epsilon$ values. It can be proven that the minimum efficiency is proportional to $W$ in the case of Carnot cycles.

To exemplify heat pumps, it suffices to exchange the values of $n_{l}$ and $n_{h}$, keeping $\epsilon_{l}$ and $\epsilon_{h}$ the same as before. Instead of efficiency, it is usual (and natural) in the case of heat pumps to define a coefficient of performance (COP) as the ratio of the amount of heat generated in the hot reservoir (e.g., in the house) to the work required to operate the system (e.g., from the power line). Thus, for reciprocal engines, the COP is the reciprocal of $\eta$. Negative work in Fig. 3 corresponds to heat pumps.

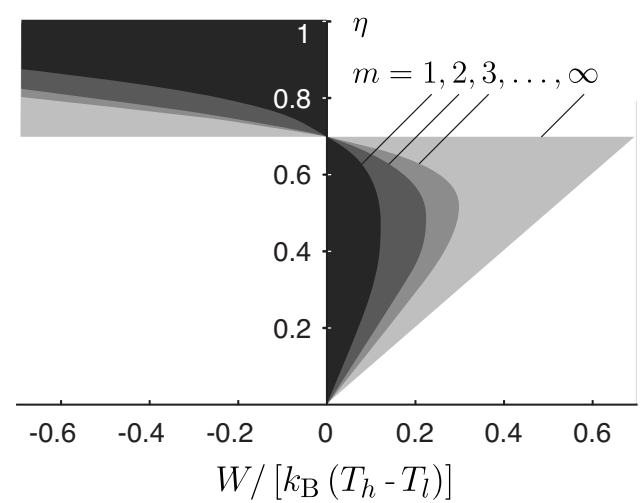

FIG. 3. Available heat-engine (right-hand side) and heat-pump (left-hand side) efficiencies as a function of the average work performed per cycle, $W$, in the case of $m=1$ (Otto cycle), $m=2, m=3$, and $m=\infty$ subreservoirs. Let us emphasize that to construct a Carnot engine out of Otto cycles, every Otto cycle must be infinitesimal. The Carnot cycles thus require an infinite number of subreservoirs. We have set $\beta_{l}=1.38, \beta_{h}=0.42$. For reversible engines the coefficient of performance (COP) of heat pumps is the reciprocal of $\eta$.

Negative temperatures. If the number of weight 1 balls in the reservoir at altitude $\epsilon=1$ is equal to 7000 and the number of upper state electrons in the reservoir at energy $\epsilon=2$ is equal to 8000 the reservoir temperatures are both negative, with $\beta_{l}=-0.69, \beta_{h}=-0.85$. It follows that the reservoir at altitude $\epsilon_{h}=2$ is the colder one, while the reservoir at altitude $\epsilon_{l}=1$ is the hotter one. The $Q$ and $W$ values are simply opposite in sign to the ones given in the previous paragraph. The system is a heat pump with some work flowing into it and heat being transferred to the hotter reservoir. If the above values of $n_{l}$ and $n_{h}$ are interchanged, the system becomes a heat engine indistinguishable from the one initially considered as far as the operating parameters are concerned.

Positive and negative temperatures. If $n_{l}=4500, n_{h}$ $=5500$, and $\epsilon_{l}=1, \epsilon_{h}=2$ as before, the temperature of one reservoir is positive while the temperature of the other reservoir is negative. The maximum attainable efficiency is in that case unity.

\section{CONCLUSION}

We have shown that quantum heat engines may be equivalent to purely mechanical engines akin to water mills. Most people would picture heat as particles moving randomly in a box, in which case heat energy is kinetic energy. However, at the other extreme, heat may be solely potential energy. This latter picture has the advantage of showing from the very beginning that the concept of time is entirely irrelevant to the understanding of Carnot cycles. In contradistinction, the concept of kinetic energy requires that the concept of time be introduced. This observation led us to the present mechanical picture, the Carnot cycle being viewed as the limit of infinitesimal Otto cycles.

Precisely, our model consists of a sequence of reservoirs at various altitudes containing balls of various weights. An infinitesimal Otto cycle consists of picking up a ball at random from a reservoir and carrying it to the next. Let us note 
that from our viewpoint, thermal engines may be distinguished from conservative devices only by the fact that an element of chance enters in the former and not in the latter. That is, as long as we have no information concerning individual balls in a bag containing $N$ balls, the probability of picking up any one ball is $1 / N$. The treatment given in the present framework is rigorous, while the introduction of temperature as is done in classical thermodynamics makes sense only in the large $N$ limit.

When the concepts of temperature and entropy are introduced according to the rules of quantum statistical mechanics, the expressions for the Carnot efficiency and work performed per cycle may be obtained. If the reservoir temperatures are both negative, the results are symmetrical to those relating to positive temperatures, heat engines, and heat pumps getting interchanged. Let us emphasize that these results were based on a simple probability law. Namely, that the probability of picking up a particular ball from an urn containing $N$ identical balls is $1 / N$. Thorough stirring of the balls in the urn before a ball is being picked up is implied, a condition that may not be fulfilled when the cycle is not slow. Many recent papers on quantum heat engines and heat pumps deal with the production of maximum power rather than maximum energy per cycle, as is done here. To investigate such questions on the basis of our model a detailed analysis of ball randomization would be needed.
[1] E. Geva and R. Kosloff, J. Chem. Phys. 96, 3054 (1992).

[2] T. Feldmann, E. Geva, R. Kosloff, and P. Salomon, Am. J. Phys. 64, 485 (1996).

[3] T. Opatrny and M. Scully, Fortschr. Phys. 50, 657 (2002).

[4] J. He, J. Chen, and B. Hua, Phys. Rev. E 65, 036145 (2002).

[5] T. D. Kieu, Phys. Rev. Lett. 93, 140403 (2004).

[6] H. T. Quan, Y.-X. Liu, C. P. Sun, and F. Nori, Phys. Rev. E 76, 031105 (2007).

[7] N. S. Carnot, Biographie et Manuscrit Publiés sous le Haut Patronage de l'Académie des Sciences (Gauthier-Villars, Paris, 1927). Most historians consider that these manuscripts were written by Carnot around 1824 .

[8] A. Kastler, Sadi Carnot et l'essor de la Thermodynamique (Editions du CNRS, Paris, 1976), Chap. L'oeuvre posthume de Sadi Carnot, p. 195.
[9] M. Zemansky and R. Dittman, Heat and Thermodynamics (MacGraw-Hill, New York, 1997).

[10] V. M. Brodiansky, Sadi Carnot (Presses Universitaires de Perpignan, Perpignan, 2006).

[11] D. Schroeder, An Introduction to Thermal Physics (AddisonWesley Publishing Company, San Francisco, 2002).

[12] P. T. Landsberg, J. Phys. A 10, 1773 (1977).

[13] J. Arnaud, L. Chusseau, and F. Philippe, Eur. J. Phys. 23, 489 (2002).

[14] J. Arnaud, L. Chusseau, and F. Philippe, e-print arXiv:quant$\mathrm{ph} / 0211072 \mathrm{v} 2$.

[15] J. Arnaud, Opt. Quantum Electron. 34, 393 (2002).

[16] H. T. Quan, P. Zhang, and C. P. Sun, Phys. Rev. E 72, 056110 (2005). 